\title{
Immunometabolic disorders in the pathogenesis of systemic lupus erythematosus
}

\author{
Kamila Tokarska ${ }^{1}$, Ireneusz Majsterek², Magda Cuchra², Anna Woźniacka ${ }^{1}$ \\ ${ }^{1}$ Department of Dermatology and Venereology, Medical University of Lodz, Lodz, Poland \\ 2Department of Chemistry and Clinical Biochemistry, Medical University of Lodz, Lodz, Poland \\ Adv Dermatol Allergol 2019; XXXVI (5): 513-518 \\ DOI: https://doi.org/10.5114/ada.2019.85251
}

\begin{abstract}
Systemic lupus erythematosus is a chronic autoimmune disease connected with complex and unclear disorders of the immune system, which causes inflammation of body tissues and internal organs. It leads to the formation of anti-nuclear antibodies (ANA) and immune complexes. Numerous immune system disorders and dysfunctions in the biochemical processes can occur in the course of the disease, and a wide range of abnormalities associated with cellular respiratory processes and mitochondrial function have been documented. The following paper presents the current understanding of the contribution of these disorders to the pathogenesis of lupus.
\end{abstract}

Key words: lupus, mitochondrial dysfunction, necrosis, mtDNA.

\section{Introduction}

The pathogenesis of systemic lupus erythematosus (SLE) is not fully understood. According to the current literature, genetic, immunological and environmental factors are responsible for its development. In addition, antinuclear antibodies, which act against the cells and tissues of the patient, also played a crucial role in SLE development [1]. They form immune complexes that destroy blood vessel walls and cause organs damage, resulting in the clinical manifestation of the disease. Small vessels located in the skin, glomeruli, brain and digestive tract are particularly vulnerable to this process. These antibodies also act as useful serological markers of the ongoing autoimmune process, being present in $95-100 \%$ of patients with SLE. The most common antibodies observed in the course of lupus are homogeneous-type anti-nuclear antibodies, i.e. autoantibodies that react with solid and extractable nuclear antigens, anti-dsDNA, anti-Sm (Smith antigen) and anti-phospholipid antibodies [2].

\section{Immune disorders}

Inappropriate immune response in SLE patients is not limited to the harmful effect of the accumulation of immune complexes. According to many authors, T lymphocytes play a significant role in the pathogenesis of
SLE $[3,4]$. Proper functioning, activation, proliferation or T-cell apoptosis play a key role in the correct response of the immune system. T-lymphocytes are responsible for regulating the amounts of reactive oxygen intermediates (ROI) and adenosine-5'-triphosphate (ATP) produced in the mitochondria [5]. In a properly functioning immune system, autoreactive T or B lymphocytes are immediately captured and subjected to a process of planned, controlled cell death known as apoptosis. In the course of this process, the cells are destroyed without harming the body and the remains known as apoptotic bodies, are subjected to phagocytosis. These mechanisms are ineffective in patients with SLE: significant disturbances occur in the subpopulation of T-suppressor and helper lymphocytes and the T lymphocytes are abnormally activated, resulting in the generation of so-called autoreactive lymphocytes. This results in an increased incidence of inflammatory and autoimmune processes [6, 7].

CD4 + T cell dysfunctions have been widely documented in patients with SLE $[8,9]$. In the course of this disease, an increase in Th17 subpopulations can be observed, which have proven pro-inflammatory effects. The first reported abnormality in T lymphocyte function was decreased interleukin 2 (IL-2) production. It plays a crucial role in the development and function of T regulatory lymphocytes. These cells are responsible for maintaining immune tolerance. Regulatory lymphocytes expressing

Address for correspondence: Kamila Tokarska MD, Department of Dermatology and Venereology, Medical University of Lodz, pl. Hallaera 1, 90-647 Lodz, Poland, phone: +48 500144 909, e-mail: kamka1990@o2.pl Received: 9.03.2018, accepted: 10.05.2018. 
CD4+Foxp3 play a key role in this phenomenon. The level of these cells has been reduced in patients with SLE [8]. Losing the tolerance to their own antigens has also been attributed to the impairment of CD4+CD25+T-lymphocytes, leading to polyclonal B cell activation and autoantibody production [9].

The disruption of CD4+T cell function is associated with abnormal cell metabolism and mitochondrial dysfunction [10]. Mitochondria, one of the organelles present in eukaryotic cells, are primary the site of ATP production as a result of cellular respiration. ATP is the source of cell energy. Mitochondria control the majority of the metabolic processes located intracellularly. Disorders of these organelles have been documented in the literature on many chronic diseases, including SLE [11]. Patients with SLE can be assigned with a mitochondrial function assessment score based on an assessment of mitochondrial membrane potential CD4+ T lymphocytes. Under physiological conditions, electrons are transported within the inner mitochondrial membrane, resulting in a release of energy, which facilitates the transport of protons from the mitochondrial matrix to the inner membrane. This process creates an electrochemical gradient known as the mitochondrial membrane potential $[\Delta \psi(\mathrm{m})]$. Maintaining this potential at physiological levels ensures proper biochemical activity, including the production of ATP and oxidative phosphorylation [12]. It is currently believed that the mitochondrial membrane potential within the lymphocytes of SLE patients is elevated. Perl et al. reported that the early phase of T-cell activation and apoptosis in lymphocytes is characterized by abnormal mitochondrial membrane potential. This condition correlates with the reduced cellular energy production in the form of ATP, electron transport disturbances, and inhibition of cellular metabolism. These phenomena exacerbate oxidative stress in cells [13].

\section{Imbalance of necrosis}

Necrosis is a form of improper cell death which results in the premature destruction of cells in normal tissue by autolysis. It can be caused by various factors such as infection, toxins, or trauma. This process results in the loss of cell membrane integrity and release of products of cell death into the extracellular space. Oxidative stress can be one of the factors that leads the cells into the necrosis pathway [14]. The necrotic cell releases a number of enzymes from broken lysosomes after the uncontrolled break-up of the organelles. These enzymes exacerbate the inflammatory response after entering the extracellular space. As a result, phagocytic cells are activated, which recognize and absorb the fragments of necrotic cells. This activation of phagocytes and dendritic cells by necrotic cells is closely related to the inhibition of the production of anti-inflammatory factors such as in terleukin 10 (IL-10), transforming growth factor $\beta$ (TGF- $\beta$ ): a state which intensifies the production of inflammatory cytokines including tumour necrosis factor $\alpha$ (TNF- $\alpha$ ), interleukin 1 (IL-1), interleukin 6 (IL-6) or interleukin 8 (IL-8) [14]. It is believed that the presence of an abnormal potential across the mitochondrial membrane favours the activation of the necrotic pathway, which correlates with the intensification of pro-inflammatory reactions in the course of SLE and its clinical manifestations [15]. The release of cellular remnants from cells, including the residues of the cell nucleus, stimulates the formation of autoantibodies such as anti-Ro and anti-dsDNA antibodies in the course of SLE resulting in blood vessel damage [16].

\section{Reactive oxygen species}

Hyperpolarization of the mitochondrial membrane can be observed along with disturbances in the $\mathrm{pH}$ of the cytoplasm of tested lymphocytes. Gergely et al. isolated circulating lymphocytes (peripheral blond lymphocytes $\mathrm{PBL}$ ) from a group of 15 patients diagnosed with SLE, as well as a group of 10 healthy individuals and 10 patients suffering from rheumatoid arthritis. Their findings indicated that isolated lymphocytes showed lupus-specific apoptotic disorders, hyperpolarization of the mitochondrial membrane, and abnormal pH in the cell cytoplasm, with the pro-apoptotic tendency of the lymphocytes of the SLE patients intensified by alkalisation of the intracellular environment [17]. By contrast, lymphocytes isolated from healthy donors and patients with rheumatoid arthritis did not reveal mentioned disorders. In addition, another important disorder observed in SLE patients was an increased level of reactive oxygen species (ROS, reactive oxygen intermediates - ROI) [17]. These chemical compounds contain oxygen atoms with an unpaired electron (radical) or O-O bonds. These substances are formed in the mitochondria, as a result of cellular respiration processes, and fulfil a number of functions among the normal course of metabolic pathways, in the pathogenesis of diseases and in the process of aging of the body [18]. Excessive production of ROS is caused by oxidative stress, defined as the imbalance between the amount of ROS and the ability of the cell to remove them effectively, with cellular ROS concentration regulated by numerous antioxidants, such as glutathione, tocopherols and antioxidant enzymes. Disturbances of the balance between prooxidative and antioxidative compounds induce oxidative stress, which can lead to cells damage taking place through apoptosis or the necrosis pathway [19].

Oxidative stress can be exacerbated in patients with SLE $[16,17,20]$. Its presence contributes to disorders of the immune response, disturbances of cell metabolic pathways, transmission on the apoptotic pathway and increase the formation of autoantibodies in the course of this disease. This in turn correlates with the severity of the symptoms related to SLEDAI score (SLE disease activity index). Abnormal ROS levels have been observed 
in lymphocytes isolated from the blood of SLE patients [17], as well as a reduced level of glutathione, which is a strong antioxidant responsible for maintaining the REDOX balance in the body $[16,20]$. Long-lasting oxidative stress in patients with SLE increase the chance of ischemic heart disease, which is one of the causes of death in this group of patients [16, 21].

\section{Respiratory chain}

One of the stages of cellular respiration is the respiratory chain, also known as the electron transport chain: a set of chemical compounds arranged according to their increasing oxidation-reduction potentials. The function of the electron transport chain and oxidative phosphorylation is the oxidation of NADH and FADH2 and the retention of released energy in the ATP molecule. The process takes place in mitochondrial internal membrane [22]. The respiratory chain consists of subunits coded by mitochondrial DNA (mtDNA). Abnormalities in the function and course of the respiratory chain have been widely documented in mitochondrial diseases: rare conditions whose disturbances can be classified as monogenic or chromosomal. Increasingly, abnormalities in mtDNA can be found in multifactorial chronic diseases associated with metabolic disorders, such as type 2 diabetes [23].

Respiratory chain abnormalities have been also documented in patients with SLE. Doherty et al. reported an increase in the activity of the respiratory chain in lymphocytes isolated from peripheral blood in SLE patients and that activity of Complex I increased within the chain [24]. Complex I called NADH dehydrogenase is present in the internal membrane of the mitochondria, which initiates a series of reactions by oxidizing NADH with the simultaneous reduction of ubiquinone (a chemical compound responsible for the transmission of electrons in the respiratory chain) present in the membrane. $\mathrm{NADH}$ dehydrogenase is responsible for the production of about $40 \%$ of the proton gradient resulting from the action of the respiratory chain, which directly correlates with the amount of ATP produced [25]. Complex I is also one of the places where a premature leakage of electrons to oxygen may occur, leading to the formation of harmful peroxides. The presence of deficiencies or inadequacies in the action of NADH dehydrogenase have been observed in diseases of the human nervous system, such as Leber's hereditary optic neuropathy and Parkinson's disease [26]. Impaired respiratory chain function at this level leads to a compensatory increase in the mass and volume of mitochondria and to increased oxidative stress in these patients.

\section{Megamitochondria}

In addition to disturbances in the mitochondrial membrane potential and a reduction in the amount of
ATP produced in the course of cellular respiration, SLE patients often demonstrate abnormalities in morphology and mitochondrial structure. Moreover, it was found that in T cells of patients with lupus more mitochondria are observed in relation to normal T cells. Furthermore, lupus lymphocytes contain several times bigger mitochondria than normal lymphocytes, called megamitochondria [27]. It turns out that the hyperpolarization of the mitochondrial membrane coincides with an increase in the mass of these organelles, resulting in the formation of socalled "megamitochondria" [10]. The mass of the organelle in this case is significantly increased and its shape is irregular; such structures are also found in alcoholic liver cells [28]. The presence of megamitochondria has been also reported in other chronic diseases such as diabetes, amyloidosis and SLE [29]. It has been demonstrated that cells displaying megamitochondria demonstrate a significantly reduced ability to synthesize ATP and hence produce energy. However, it has been proven that the removal of excess free radicals from cells promote the return of mitochondria to their normal size. A sudden increase in the amount of oxygen radicals increases the oedema of the mitochondria, leading to the formation of abnormal organelles, which subsequently activates caspase and leads to condensation of the nucleus DNA. As a result, the cell containing megamitochondria enters the apoptotic pathway [29]. Further research is required to determine whether the formation of megamitochondria is the cause or the result of disorders in the cell and the extent to which their presence leads to the clinical manifestation of the disease.

\section{Oxidative mtDNA damage and mitochondrial antioxidant enzymes}

Mitochondrial DNA (mtDNA) is genetic material in the form of a circular chain located in the matrix of the mitochondrion. A single human mitochondrion contains four to ten circular DNA molecules with a length of 16569 base pairs; each of which encodes 37 genes: thirteen of them coding proteins, 22 coding tRNA, and the last two rRNA. Although the mtDNA encodes part of the mitochondrial respiratory chain proteins, the nuclear genome encodes most of the proteins included in its composition [30].

A growing number of evidence has shown that mitochondrial dysfunction may be associated with the pathogenesis of the following diseases: diabetes, neurodegenerative diseases and autoimmunity disorders such as SLE. It was shown that the disturbed mitochondrial function is associated with elevated generation of ROS, increased transmembrane potential, decreased ATP generation and greater amount of mitochondria. These changes may result in abundance of mtDNA (mitochondrial DNA) [15]. Disorders within mtDNA can be divided into two groups. The first includes congenital mitochondrial diseases, which are usually inherited in the maternal line, 
e.g. Leber's hereditary optic neuropathy [2]. The second group comprises a number of diseases demonstrating the mitochondrial dysfunction and mtDNA abnormalities, e.g. lupus; however, these mitochondrial faults are the only risk factors for the development of the disease. Considering the proven role of mitochondrial disorders in the pathogenesis of lupus, it seems reasonable to search for abnormalities in the mtDNA of SLE patients. Jönsen et al. demonstrated a relationship between the presence of mtDNA polymorphisms and susceptibility to SLE [31].

What is interesting, mtDNA is more prone to ROS activity than nucleus DNA, especially in the D310 region, because of lack of introns and histone protection, insufficient DNA repair mechanisms and its localization. Lin et al. indicated a lower number of mtDNA copies and greater heteroplasmatic change in D310 region in patients with SLE. It is suggested that some changes in mtDNA structure such as: 4997 bp deletion, D310 variants harbouring 1 to 6 bp insertion or deletion, can interfere with homoplasmy to heteroplasmy [32].

Lee et al. found that the appearance of a 4977 bp deletion in the leukocyte mtDNA is associated with a lower level of IL-10 and anti-inflammation cytokine in plasma of SLE patients. They postulated that this kind of alternation in mtDNA may be a useful biological marker of severity of the discussed disease. Moreover, in a group of SLE patients with a more advanced stage of the disease, it was observed that frequency of leukocyte D310 heteroplasmy was higher and the copy number of leukocyte mtDNA was lower. They also postulated that changes in D310 region correlate with organ injures such as lupus nephritis. This group of investigators strongly underlines that D310 heteroplasmy of mtDNA is an excellent biomarker for observing the progression of SLE as well as a risk of lupus nephritis [33].

Altered mtDNA may influence changes in energy metabolism and tissue dysfunction [34]. This group of researchers also found that the number of mtDNA copies correlate with a level of 8-oxoG. They found that a decreased number of mtDNA copies is connected with an elevated level of 8-oxoG [35]. Numerous studies confirm an increased level of 8-oxoG, oxidative stress marker, in blood samples from patients with SLE [33]. Abundance in the number of leucocyte mtDNA copies may be also associated with cripple biosynthesis of mitochondria [36]. This finding may suggest the role of oxidative stress in SLE development [35]. Despite these findings, the role of oxidative stress in SLE pathogenesis has not been fully understood yet [36].

What is interesting, Lopez-Lopez et al. have observed an elevated level of mtDNA damage and mtDNA depletion in a group of patients with SLE in relation to healthy controls. Moreover, they noticed the relationship between the level of mtDNA damage and disease duration. This group of researchers conducted studies which correlated the level of mtDNA lesions in two groups of SLE patients. They found that the group of SLE patients with organ involvement has lower levels of mtDNA damage than the group with no major organ distress. They explain these findings as follows: the elevated level of mtDNA lesions may prompt apoptosis of SLE patients with major organ involvement, which cause a lower detection of mtDNA damage [37]. Furthermore, oxidative damage may lead to single strand breaks in the mtDNA, which induce the apoptosis process. Tann et al. postulated that accumulation of single strand breaks in mtDNA may lead to alter the mitochondrial function as well as apoptosis [38]. It is suggested that the crucial role in SLE pathogenesis may be played by mtDNA damage caused by oxidative stress [37]. Lee et al. found the relationship between the elevated level of oxidative lesions and the lower expression of mRNA of hOGG1 gene and the genes encoding anti-oxidant enzymes, proteins responsible for mitochondria biogenesis and glycolytic enzymes in leucocytes. Moreover, the literature reports that in a group of patients with SLE, not only a higher level of oxidative stress but also lower anti-oxidant capacity is observed [39].

GPX-4 is the main mitochondrial antioxidant enzyme. Some studies indicated a lower level of its expression in a group of SLE patients in relation to healthy controls. What is interesting, the level of mitochondrial GPx-4 did not change in response to the increase level of 8-oxoG [40]. Moreover, it is suggested that GPx-4 may be a good therapeutic strategy for patients with SLE by improving its expression [41]. It was proved that mitochondrial and cytosol isoforms of superoxide dismutase (SOD) enzymes play first defence lines against ROS from mitochondrial or other sources. This metalloproteinase catalyses dismutation of superoxide radical into oxygen and hydrogen peroxide. In human cells we can find three isoforms of this antioxidant enzyme: SOD 1 is localized in the cytoplasm, SOD2 has mitochondrial localization (MnSOD) and SOD3 - extracellular. What is interesting, it was proved that the antibody to SOD is responsible for enzyme inactivation, which may cause accumulation of oxidative DNA damage in SLE patients. Numerous studies found a lower level of both forms of SOD enzyme in a group of patients with SLE in relation to healthy controls [42, 43]. A study conducted on a mice model with SLE also showed a significantly higher expression of MnSOD in the rental cortex. Authors explained this fact by stating that the increased level of renal cortical MnSOD may be a compensatory change in reaction to the increased level of oxygen stress. Furthermore, they also suggested that oral supplementation of antioxidants may lead to reduced renal oxidative stress [44]. Also, it is postulated that presence of $47 C>T$ polymorphism of MnSOD gene may influence the enzyme activity. This polymorphic variant of MnSOD is localized on codon 16 and is responsible for Ala/Val substitution at -9 in mitochondrial targeting sequence (MTS) of mature protein. It is suggested that 
presence of this substitution may lead to a change in the secondary structure of the MTS, which may have consequences in delivery of MnSOD to the mitochondria [45]. The study conducted by Yen et al. did not indicate any relationship between the presence of this polymorphic variant of MnSOD gene in Taiwan population [46]. Similar research was conducted by a Polish team. Despite a lack of association between the presence of this polymorphic variant and SLE development, Sobkowiak et al. postulated that Val/Val genotype of MnSOD gene may be associated with some clinical manifestation in patients with SLE. They indicated that Val/Val genotype may contribute to the immunologic manifestation [47].

\section{Conflict of interest}

The authors declare no conflict of interest.

\section{References}

1. Klonowska-Szymczyk A, Robak E. Współczesne poglądy na etiopatogenezę układowego tocznia rumieniowatego. Postep Hig Med Dosw 2011; 65: 683-703.

2. Cozzani E, Drosera M, Gasparini G, Parodi A. Serology of lupus erythematosus: correlation between immunopathological features and clinical aspects. Autoimmune Dis 2014; 2014: 321359.

3. Hoffman RW. T cells in the pathogenesis of systemic lupus erythematosus. Front Biosci 2001; 6: D1369-78.

4. Rastin M, Mahmoudi M, Hatef M, et al. T lymphocyte apoptosis in systemic lupus erythematosus patients. Iran J Basic Med Sci 2013; 16: 936-41.

5. Perl A, Gergely P Jr, Banki K. Mitochondrial dysfunction in T cells of patients with systemic lupus erythematosus. Int Rev Immunol 2004; 23: 293-313.

6. Rahman A, Isenberg DA. Systemic lupus erythematosus. N Engl J Med 2008; 358: 929-39.

7. Tsokos GC. Systemic lupus erythematosus. N Engl J Med 2011; 365: 2110-21.

8. Konya C, Paz Z, Tsokos GC. The role of T cells in systemic lupus erythematosus: an update. Curr Opin Rheumatol 2014; 26: 493-501.

9. Krajewska M, Weyde W, Klinger M. The signifi cance of regulatory CD4+CD25+ T cells in the pathogenesis and treatment of kidney disease. Postepy Hig Med Dosw (Online) 2007; 61: 178-84.

10. Morel L. Immunometabolism in systemic lupus erythematosus. Nat Rev Rheumatol 2017; 13: 280-90.

11. Nicolson GL. Mitochondrial dysfunction and chronic disease: treatment with natural supplements. Integr Med 2014; 13 : 35-43.

12. Skulachev VP. Transmembrane electrochemical H+-potential as a convertible energy source for the living cell. FEBS Lett 1977; 74: 1-9.

13. Perl A, Nagy G, Gergely P, et al. Apoptosis and mitochondrial dysfunction in lymphocytes of patients with systemic lupus erythematosus. Methods Mol Med 2004; 102: 87-114.

14. Paduch R, Klatka M, Klatka J. Types of cell death. Pomeran J Life Sci 2015; 61: 411-8.

15. Gergely P Jr, Grossman C, Niland B, et al. Mitochondrial hyperpolarization and ATP depletion in patients with systemic lupus erythematosus. Arthritis Rheum 2002; 46: 175-90.
16. Perl A. Oxidative stress in the pathology and treatment of systemic lupus erythematosus. Nat Rev Rheumatol 2013; 9: 674-86.

17. Gergely P Jr, Niland B, Gonchoroff N, et al. Persistent mitochondrial hyperpolarization, increased reactive oxygen intermediate production, and cytoplasmic alkalinization characterize altered IL-10 signaling in patients with systemic lupus erythematosus. J Immunol 2002; 169: 1092-101.

18. Bartosz G. Druga twarz tlenu. Wydawnictwo Naukowe PWN, Warsaw 2008.

19. Sroka J, Madeja Z. Udział reaktywnych form tlenu i reduktazy tioredoksyny w regulacji migracji komórek. Postep Biochem 2009; 55: 145-52.

20. Perl A, Gergely P Jr, Nagy G, et al. Mitochondrial hyperpolarization: a checkpoint of T-cell life, death and autoimmunity. Trends Immunol 2004; 25: 360-7.

21. Trager J, Ward MM. Mortality and causes of death in systemic lupus erythematosus. Curr Opin Rheumatol 2001; 13: 345-51.

22. Jonckheere Al, Smeitink JA, Rodenburg RJ. Mitochondrial ATP synthase: architecture, function and pathology. J Inherit Metab Dis 2012; 35: 211-25.

23. Brągoszewski P, Ostrowski J. Mitochondrial medicine. Postep Nauk Med 2009; 2: 138-48.

24. Doherty E, Oaks Z, Perl A. Increased mitochondrial electron transport chain activity at complex I is regulated by $\mathrm{N}$-acetylcysteine in lymphocytes of patients with systemic lupus erythematosus. Antioxid Redox Signal 2014; 21: 56-65.

25. Hinchliffe P, Sazanov LA. Organization of iron-sulfur clusters in respiratory complex I. Science 2005; 5735: 771-4.

26. Schapira AH. Human complex I defects in neurodegenerative diseases. Biochim Biophys Acta 1998; 1364: 261-70.

27. Nagy G, Perl A. The role of nitric oxide in abnormal T cell signal transduction in systemic lupus erythematosus. Clin Immunol 2006; 118: 145-51.

28. Chedid A, Mendenhall CL, Tosch T, et al. Significance of megamitochondria in alcoholic liver disease. Gastroenterology 1986; 90: 1858-64.

29. Wakabayashi T. Megamitochondria formation - physiology and pathology. J Cell Mol Med 2002; 6: 497-538.

30. Krishnan KJ, Turnbull DM. Mitochondrial DNA and genetic disease. Essays Biochem 2010; 47: 139-51.

31. Jönsen A, Yu X, Truedsson L et al. Mitochondrial DNA polymorphisms are associated with susceptibility and phenotype of systemic lupus erythematosus. Lupus 2009; 18: 309-12.

32. Lin CS, Lee HT, Lee M, et al. Role of mitochondrial DNA copy number alteration in human renal cell carcinoma. Int I Mol Sci 2016; 17: E814.

33. Lee HT, Lin CS, Chen WS, et al. Leukocyte mitochondrial DNA alteration in systemic lupus erythematosus and its relevance to the susceptibility to lupus nephritis. Int J Mol Sci 2012; 13: 8853-68.

34. Vyshkina T, Sylvester A, Sadiq S, et al. Association of common mitochondrial DNA variants with multiple sclerosis and systemic lupus erythematosus. Clin Immunol 2008; 129: 31-5.

35. Lee HT, Wu TH, Lin CS, et al. Oxidative DNA and mitochondrial DNA change in patients with SLE. Front Biosci 2017; 22: 493-503.

36. Lee HC, Wei YH. Mitochondrial biogenesis and mitochondrial DNA maintenance of mammalian cells under oxidative stress. Int J Biochem Cell Biol 2005; 37: 822-34.

37. Lopez-Lopez L, Nieves-Plaza M, Castro Mdel R, et al. Mitochondrial DNA damage is associated with damage accrual 
and disease duration in patients with systemic lupus erythematosus. Lupus 2014; 23: 1133-41.

38. Tann AW, Boldogh I, Meiss G, et al. Apoptosis induced by persistent single-strand breaks in mitochondrial genome: critical role of EXOG (5'-EXO/endonuclease) in their repair. J Biol Chem 2011; 286: 31975-83.

39. Lee HT, Lin CS, Lee CS, et al. Increased 8-hydroxy-2'-deoxyguanosine in plasma and decreased mRNA expression of human 8-oxoguanine DNA glycosylase 1, anti-oxidant enzymes, mitochondrial biogenesis-related proteins and glycolytic enzymes in leucocytes in patients with systemic lupus erythematosus. Clin Exp Immunol 2014; 176: 66-77.

40. Liang H, Van Remmen H, Frohlich V, et al. Gpx4 protects mitochondrial ATP generation against oxidative damage. Biochem Biophys Res Commun 2007; 356: 893-8.

41. Lai ZW, Hanczko R, Bonilla E, et al. N-acetylcysteine reduces disease activity by blocking mammalian target of rapamycin in T cells from systemic lupus erythematosus patients: a randomized, double-blind, placebo-controlled trial. Arthritis Rheum 2012; 64: 2937-46.

42. Shah D, Aggarwal A, Bhatnagar A, et al. Association between $T$ lymphocyte sub-sets apoptosis and peripheral blood mononuclear cells oxidative stress in systemic lupus erythematosus. Free Radic Res 2011; 45: 559-67.

43. Shah D, Mahajan N, Sah S, et al. Oxidative stress and its biomarkers in systemic lupus erythematosus. J Biomed Sci 2014; 21: 23.

44. Mathis KW, Venegas-Pont M, Masterson CW, et al. Oxidative stress promotes hypertension and albuminuria during the autoimmune disease systemic lupus erythematosus. Hypertension 2012; 59: 673-9.

45. Rosenblum JS, Gilula NB, Lerner RA. On signal sequence polymorphisms and diseases of distribution. Proc Natl Acad Sci USA 1996; 93: 471-3.

46. Yen JH, Chen CJ, Tsai WC, et al. Cytochrome P450 and manganese superoxide dismutase genes polymorphisms in systemic lupus erythematosus. Immunol Lett 2003; 90: 19-24.

47. Sobkowiak A, Lianeri M, Wudarski M, et al. Manganese superoxide dismutase Ala-9Val mitochondrial targeting sequence polymorphism in systemic lupus erythematosus in Poland. Clin Rheumatol 2008; 27: 827-31. 